\title{
Importância da avaliação oftalmológica em recém-natos
}

\author{
Importance of the ophthalmological evaluation in newborns
Daniel Wasilewski ${ }^{1}$, Rommel J. Zago ${ }^{2}$, Anne M.C. Bardal ${ }^{2}$, Ticiano M. Heusi ${ }^{2}$, Flávia P. Carvalho², Lázara F. Maciel², Hamilton Moreira ${ }^{3}$, Marcelo L. Gehlen ${ }^{4}$, Evanguelia A. Shwetz ${ }^{5}$

\section{Resumo}

Objetivo: verificar a existência de afecções oculares, nas primeiras 48 horas de vida de recém-natos, e relacioná-las com a suspeita clínica dos pediatras.

Métodos: realizou-se um estudo prospectivo em que todos os recém-natos no período de julho a dezembro de 2000 foram examinados no alojamento conjunto do Hospital Universitário Evangélico de Curitiba (HUEC). Seiscentos e sessenta e sete recém-natos foram avaliados, através de um protocolo, por médicos residentes e preceptores de oftalmologia, independentemente se havia ou não qualquer suspeita de alteração ocular pelo pediatra. Procedeu-se à inspeção, iluminação oblíqua, avaliação de desvio ocular e oftalmoscopia direta à distância (reflexo vermelho) em todos os pacientes.

Resultados: em 3,75\% dos pacientes avaliados, encontrou-se alguma alteração ocular. A principal afecção ocular foi a opacidade corneana, detectada pelo exame de reflexo vermelho à distância. Cinqüenta e seis por cento dos recém-natos portadores de patologia ocular passaram despercebidos pelos pediatras, neonatologistas e pais.

Conclusão: este estudo demonstrou que a maioria das desordens oculares presentes ao nascimento não é diagnosticada pelos pediatras $(56 \%)$, evidenciando, desta forma, a importância do exame oftalmológico como rotina no atendimento ao recém-nato nas primeiras 48 horas de vida.

J Pediatr (Rio J) 2002; 78 (3): 209-12: recém-nascidos, ambliopia, leucocoria, opacidades corneanas.

\begin{abstract}
Objective: to verify the existence of ocular diseases in the first 48 hours of life of newborns and relate it to the clinical suspicious of pediatricians.

Methods: a prospective study was performed. All infants that were born between July and December of 2000 were evaluated in the nursery of Hospital Universitário Evangélico de Curitiba (HUEC). Six hundred sixty-seven newborns were evaluated through a protocol by residents and tutors of ophthalmology, regardless of pediatricians' suspicious of ocular disorder. The examination consisted of inspection, oblique illumination, evaluation of ocular deviation and direct ophthalmoscopy (red reflex) in all patients.

Results: among all examined newborns, 3.75\% showed some ocular disease. The most frequent problem was corneal opacity. The ocular diseases were not noticed by pediatricians, neonatologists and parents in fifty-six per cent of the cases presenting some problem.

Conclusions: this study showed that many ocular disorders presented at birth are not noticed by pediatricians (56\%), which highlights the importance of ophthalmological evaluation in all newborns as a routine examination in the first 48 hours of life.
\end{abstract}

J Pediatr (Rio J) 2002; 78 (3): 209-12: newborns, amblyopia, leukocoria, corneal opacities.

\section{Introdução}

A oftalmologia pediátrica não envolve somente o tratade Curitiba, PR (HUEC).

2. Médicos residentes de Oftalmologia - HUEC.

3. Chefe do Serviço de Oftalmologia - HUEC.

4. Preceptor do Serviço de Oftalmologia - HUEC.

5. Chefe do Serviço de Neonatologia - HUEC.

Artigo submetido em 08.08.01, aceito em 27.02.02. mento de doenças oculares em crianças, mas, também, seus efeitos no sistema visual imaturo ${ }^{1}$.

Várias afecções oculares infantis, algumas delas com alto índice de morbi-mortalidade, manifestam-se clinica- 
mente nos primeiros dias, ou até nas primeiras horas de vida. Todavia, alterações oculares simples, e muitas vezes graves, podem passar despercebidas por pediatras, neonatologistas e pelos pais do recém-nato. A leucocoria (pupila branca), um dos sinais clínicos mais evidentes, pode ser devido a várias causas, geralmente graves, como catarata congênita, persistência de vítreo primário hiperplásico, retinopatia da prematuridade e outras, que, se não forem diagnosticadas e tratadas precocemente, contribuirão para um prognóstico visual bastante reservado ${ }^{1}$.

Não são somente as doenças graves que podem causar dano visual importante. Alterações oculares, que podem parecer simples, também aparecem como grande causa de ambliopia (diminuição da acuidade visual, quase sempre unilateral, que ocorre em crianças visualmente imaturas devido à falta de uma imagem nítida na retina ${ }^{2}$ ), como o caso do estrabismo congênito ${ }^{3}$.

Este estudo foi realizado com o objetivo de verificar a existência de alterações oculares em recém-natos (RNs) e relacioná-las com a suspeita clínica dos pediatras.

\section{Pacientes e métodos}

Foram avaliados 667 recém-natos no período de julho a dezembro de 2000, no Hospital Universitário Evangélico de Curitiba (HUEC). Foi instituído um protocolo no qual constavam as seguintes informações: idade do RN, idade gestacional, peso ao nascimento, tipo de parto, patologias e medicações durante a gestação, avaliação de desvio ocular, iluminação oblíqua, reflexo fotomotor e inspeção à oftalmoscopia direta (reflexo vermelho). Reflexo vermelho é o reflexo retiniano de coloração avermelhada, visto com o oftalmoscópio através da pupila do examinando, desde que os meios oculares (córnea, humor aquoso, cristalino e humor vítreo) estejam transparentes.
Os critérios de inclusão foram os seguintes: recémnatos a termo, sadios, nascidos entre $1^{\circ}$ de julho e $1^{\circ}$ de dezembro de 2000, presentes no alojamento conjunto nas primeiras 48 horas de vida. Os critérios de exclusão foram: presença de qualquer tipo de malformação congênita e doenças ao nascimento.

Os recém-natos foram examinados no alojamento conjunto, dentro das primeiras 48 horas de vida, por médicos residentes do primeiro ano, e supervisionados pelos preceptores de oftalmologia do HUEC. Os que apresentaram alguma alteração ao primeiro exame foram submetidos a um exame oftalmológico completo no ambulatório de oftalmologia, incluindo tonometria e oftalmoscopia indireta com dilatação pupilar. A avaliação oftalmológica foi realizada independentemente se havia ou não suspeita clínica de alteração ocular pelo pediatra.

Este estudo foi aprovado pelo Comitê de Ética em Pesquisa da Instituição.

\section{Resultados}

Vinte e cinco dos 667 recém-natos avaliados, correspondendo a $3,75 \%$, apresentaram alterações oculares que, posteriormente, foram confirmadas através de um exame oftalmológico completo. Os demais pacientes apresentaram-se normais à primeira avaliação oftalmológica.

As alterações oculares mais encontradas foram: opacidades corneanas -8 casos (32\%), desvio ocular convergente -6 casos (24\%), conjuntivite neonatal -5 casos $(20 \%)$, hiposfagma (sangramento subconjuntival) - 2 casos (8\%), catarata congênita unilateral -2 casos $(8 \%)$, anisocoria -1 caso (4\%), e coloboma de íris e coróide -1 caso (4\%) (Figura 1).

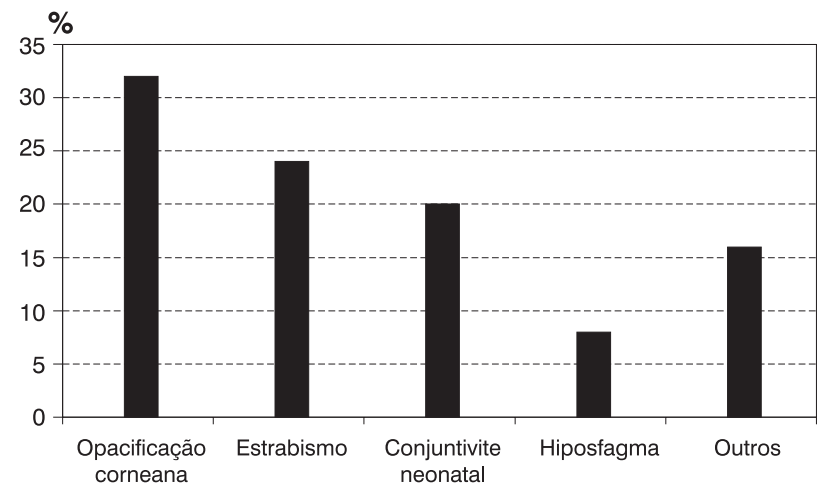

Figura 1 - Freqüência das alterações oculares 
Todos os casos de opacidade corneana foram causados aparentemente pelo trauma do parto. Nenhum caso de conjuntivite teve necessidade de coleta de material antes do início do tratamento, não sendo possível estabelecer o diagnóstico etiológico da infecção. Todos os RNs com alteração ao primeiro exame tiveram essas alterações confirmadas pelo segundo exame oftalmológico, realizado no ambulatório de oftalmologia.

Dos recém-natos que apresentaram alterações oculares ao exame primário, 14 deles, correspondendo a 56\%, não tiveram essas alterações percebidas pelos pediatras. Nesses 14 RNs, houve 5 casos de opacidade corneana, 3 casos de estrabismo convergente, 2 casos de conjuntivite neonatal, os 2 casos de catarata congênita, o único caso de anisocoria e o único caso de coloboma de íris e coróide.

\section{Discussão}

A maior diferença entre a oftalmologia pediátrica e a do adulto é o impacto que as desordens oculares podem causar ao desenvolvimento visual da criança ${ }^{1}$. Recentes pesquisas têm demonstrado que a avaliação oftalmológica precoce em recém-natos propicia um melhor desenvolvimento visu$\mathrm{al}^{4}$. De acordo com von Noorden, 2 a $4 \%$ das crianças americanas têm ambliopia, causada principalmente pela anisometropia (larga diferença refrativa entre os dois olhos ${ }^{5}$ ) e estrabismo ${ }^{6}$. Segundo Ehrlich e cols., aproximadamente $80 \%$ das crianças em idade pré-escolar nos Estados Unidos não são triadas para desordens oculares ${ }^{7}$. Não há motivo para uma medicina preventiva tão inadequada, pois os exames de investigação não têm custo, são relativamente de fácil realização e requerem um tempo bastante curto. E para evitar perda visual permanente, uma investigação padronizada deveria ser incorporada ao exame de crianças aparentemente sadias, para que o tratamento, se necessário, possa ser iniciado a tempo 5 .

Ambliopia e estrabismo são doenças pediátricas comuns e, se não forem detectadas precocemente, podem levar à perda de visão binocular e até à cegueira funcional. Catarata e glaucoma congênitos são raros, mas podem desenvolver perda visual significante se não tratados imediatamente. Retinoblastoma, se não tratado, pode causar cegueira e ser fatal. Todas essas doenças podem ser controladas ou potencialmente curadas se forem detectadas precocemente ${ }^{5}$.

O aparecimento da leucocoria na persistência do vítreo primário hiperplásico é precoce (ao nascer) ${ }^{8}$, devendo entrar no diagnóstico diferencial.

Leucocoria é um sinal clínico que indica a opacidade do cristalino e/ou do segmento posterior do olho (vítreo e retina), por isso a pupila se torna esbranquiçada, não sendo possível a visualização do reflexo vermelho.

Opacidade corneana é a diminuição da total transparência da córnea, devido a lesões em uma ou mais das cinco camadas que a compõem. No caso do traumatismo de parto, ocorre descompensação corneana devido a uma quebra na membrana de Descemet.

Trabalhos demonstram um maior risco de retinopatia, estrabismo e ambliopia nos RNs, especialmente naqueles com baixo peso 9 .

A Associação Americana de Oftalmologia e Estrabismo e a Academia Americana de Pediatria orientam que o exame oftalmológico para os recém-natos, tanto prematuros como os de termo, deve ser composto de: exame de reflexo vermelho (através da oftalmoscopia direta à distância), inspeção, resposta pupilar e observação de desvio ocular, e orienta que esta avaliação deve ser obrigatória em todas as crianças com peso ao nascimento menor que $1.500 \mathrm{~g}$ e naquelas com história familiar de anormalidades oculares, como catarata congênita e retinoblastoma, já que $60 \%$ destes apresentam leucocoria ${ }^{10}$.

Este estudo mostra que o exame oftalmológico nas primeiras 48 horas de vida deve ser realizado também nos RNs aparentemente sadios, podendo ser feito por qualquer integrante da equipe médica treinado, inclusive auxiliares e técnicos de enfermagem.

Concordando com a literatura ${ }^{7}$, neste estudo, apenas a minoria (44\%) dos RNs com patologias oculares foi avaliada a pedido da equipe pediátrica.

O estudo demonstrou que $56 \%$ das desordens oculares presentes ao nascimento não foram diagnosticadas pelos neonatologistas e pediatras, evidenciando-se a importância da utilização do exame oftalmológico como rotina no atendimento ao recém-nascido, pois a facilidade do primeiro exame, que pode ser realizado por qualquer integrante da equipe médica, leva a considerar que sua falta pode, além de prejudicar profundamente a vida futura do paciente, ser considerada omissão médica.

\section{Referências bibliográficas}

1. Olitsky SE, Nelson LB. Common ophthalmologic concerns in infants and children. Pediatr Clin North Am 1998; 45:993-1012.

2. Anderson RL, Baumgarten AS. Amblyopia in ptosis. Arch Ophthalmol 1980; 98:1068-72.

3. Vaughan DG, Asbury T, Riordan-Eva P. Oftalmologia Geral. $4^{\text {a }}$ ed. São Paulo: Atheneu; 1997.

4. Ryan JB. Pediatric primary care vision examination. Optom Clin 1996; 5:1-34.

5. Essman SW, Essman TF. Screening for pediatric eye disease. Am Fam Physician 1992; 46:1243-52.

6. von Noorden GK. Burian-von Noorden's Binocular vision and ocular motility: theory and management of strabismus. $3^{\mathrm{a}} \mathrm{ed}$. St. Louis: Mosby; 1985.

7. Ehrlich MI, Reinecke RD, Simons K. Preschool vision screening for amblyopia and strabismus. Programs, methods, guidelines. Surv Ophthalmol 1983; 28:145-63. 
8. Gonçalves EA, Araújo HA, Carletti JR, Gonçalves JO. Leucocorias na clínica oftalmológica do HGV. Arquivos Brasileiros de Oftalmologia 1988; 51:77-9.

9. Hoyt CS, Nickel BL, Billson F. An ophthalmological examination of the infant. Development aspects. Surv Ophthalmol 1982; 26:177-89.

10. Romano PE. Advances in vision and eye screening: screening at six months of age. Pediatrician 1990; 17:134-41.
Endereço para correspondência:

Dr. Daniel Wasilewski

Rua Emiliano Perneta, no 680 - cj. 1608

CEP 80420-080 - Curitiba, PR

E-mail: danielwasilewski@hotmail.com 